\title{
Cuestionamientos y alternativas de respuesta al desarrollo y su expresión local
}

Riera Vázquez, C. Celia M.

Universidad Central "Marta Abreu"

de Las Villas, Cuba

celiam@uclv.edu.cu
Fabré Machado, Idalsis

Universidad Central "Marta Abreu"

de Las Villas, Cuba

idalsisFM@uclv.edu.cu

\section{Resumen}

Repasando los asideros conceptuales y metodológicos de las diversas adjetivaciones dadas al desarrollo, es ostensible la ausencia de una formulación conceptual que denote y connote el carácter esencialmente contradictorio del proceso mismo. Se pasa por alto que dicha contradicción, es condición necesaria para la existencia, movimiento y transformación de cualquier sistema $y$, por tanto, cuando se habla de desarrollo se impone destacar que su contradictoriedad se ancla en el proceso de enajenación social y la potencialidad de este estado para transformarse en su contrario. Trabajo vs capital media la comprensión y las apuestas por el desarrollo. Tal reconocimiento sitúa al desarrollo y su expresión local en la concepción que centra y enfatiza la idea de autodesarrollo articulado con lo comunitario, núcleo de la comprensión marxista de la sociedad y la historia. Co-responsables de diseminar tal reconocimiento son los intelectuales y profesores desde las cátedras, los espacios de asesoramiento, de monitoreo y decisores.

Palabras clave: desarrollo humano; lo comunitario; lo local; contradicción; emancipación;

\section{Abstract}

Reviewing the conceptual and methodological handles of the various adjectives given to development, the absence of a conceptual formulation denoting and connoting the essentially contradictory nature of the process itself is ostensible. It is overlooked that this contradiction is a necessary condition for the existence, movement and transformation of any system and, therefore, when it comes to development, it is necessary to emphasize that its contradictoriness is anchored in the process of social alienation and the potentiality of this been to become its opposite. Work vs. capital means understanding and betting on development. Such recognition places development and its local expression in the conception that focuses and emphasizes the idea of self-development articulated with the community, core of the Marxist understanding of society and history. Co-responsible for disseminating such recognition are the intellectuals and professors from the chairs, the advisory, monitoring and decision-making spaces.

Keywords: human development; the community; the local; contradiction; emancipation; 
Para reconocer las opciones, es necesario pensar a la realidad desde la perspectiva de lo objetivamente posible. Para ello debemos enriquecer nuestra visión de ella, pese a que esto suponga trascender los encuadres teóricos disponibles o las experiencias acumuladas.

Hugo Zemelman

\section{DISYUNCIONES Y DISYUNTIVAS}

\section{DEL DESARROLLO COMO}

\section{TÉRMINO}

Existe una importante dispersión terminológica respecto al desarroIlo, enmarcada en tres aspectos fundamentales -el signo, significante y significado- que, mediados por un proceso al que Merton (2002) calificó como "(...) de alienación y estereotipación recíproca (...)" representan disyunciones de unos frente a otros pretendiendo dilucidar las funciones e, incluso, la propia viabilidad de dicho proceso.

Tales aspiraciones de generalización se verifican en los actuales estudios sobre el tema desarrollo, cuya amplia presencia en los deba- tes académicos trasluce los sesgos ideologizantes desde los cuales se le aborda. Se impone como necesidad deslindar las propuestas que bajo diferentes denominaciones, son expresiones de la lógica de la dominación imperial, del paradigma de la globalización vinculadas al desarrollismo y la tecnofilia, de aquella reflexión crítica, en proceso de legitimación, gestada emergentemente en torno a las prácticas transformadoras de los movimientos populares, como opción frente al desarrollo capitalista.

Lo anterior presenta la comprensión del desarrollo como un proceso histórico-universal que debe ir orientado a la recuperación de la esencia humana, a la desalienación, a la emancipación del hombre (Marx y Engels, 1975) por tanto no puede ser concebido como un proceso de ensamblaje de dimensiones y características sino comprendido en su esencia, como relación social; consideración cardinal para el logro del ideal humanista de los proyectos emancipadores, anticapitalistas. 
Tal y como afirma M. Féliz "La idea de desarrollo vino a apuntalar políticas orientadas a promover la forma esencial del desenvolvimiento de las relaciones sociales capitalistas"1 (2015:31).

El vocablo desarrollo comienza a utilizarse como término político y posteriormente académico en 1941, en la Primera Declaración InterAliada y en la Carta del Atlántico de ese mismo año. Posteriormente se ratifica en la Conferencia de San Francisco en 1945, de la cual surgió la Organización de Naciones Unidas (Pérez Díaz, 2010).

Desde finales de los años 60 del siglo XX, el desarrollo se concebía como crecimiento económico y bienestar utilizando como instrumento de medición el Producto Interno Bruto (PIB) (Becerra y Pino, 2005). Esto es expresión de una visión cuantitativista y unilateral en la con- cepción del desarrollo, permitiendo la clasificación de los países en desarrollados o sub desarrollados.

Alrededor de la década de los setenta del siglo en cuestión, el criterio de medición incorpora aquellas condiciones propiciadoras del desarrollo del potencial humano, de ahí que se le agregó al desarrollo la cualidad de humano, en defensa de una forma de distribución más equitativa.

El fracaso de las políticas macroeconómicas para darle solución a los problemas existentes entre los setenta y los ochenta del siglo $X X$, enmarcado en la crisis estructura ${ }^{2}$ del sistema capitalista, generó una mirada alternativa a la predominante a partir de lo cual emergen las de naturaleza microeconómicas orientadas a la descentralización y la apertura a fórmulas regionales y locales que tienen al mercado como regulador por excelencia del entra-

1. En términos de apariencia, el crecimiento económico, el aumento en la productividad del trabajo y el incremento en el empleo asalariado se convirtieron en los valores fundamentales de las nuevas estrategias que apelaban a la planificación y promoción estatal del proceso de reproducción capitalista. La esencia de esas políticas era ampliar las relaciones capitalistas de producción, la expansión del capital y la mercantilización general de las relaciones sociales hacia los fines de la valorización a escala ampliada del capital. (Féliz, 2017:33)

2. La crisis estructural del Capitalismo de estos años fue sincrónica y se manifestó a través de: la crisis energética, la crisis de la deuda, la crisis alimentaria y la crisis ecológica. 
mado socioeconómico (Pérez Díaz, 2010). En tal racionalidad se encuentran distintas adjetivaciones que han acompañado al término desarrollo.

Desde esquemas y perspectivas muy disímiles, con plataformas en ocasiones divergentes el desarrollo se impone como un fin en sí mismo, más que como un proceso dialéctico. Esto marca la lógica fragmentadora del pensamiento social, que si bien ha encontrado en el desarrollo un núcleo articulador respecto a la hegemonía positivista, reproduce sus miradas desde los estancos disciplinares, que están latentes en el análisis (Riera et al, 2017).

A partir de las voces autorizadas de importantes autores cubanos y latinoamericanos así como los estudios realizados por las autoras se puede juzgar que los debates sobre el desarrollo son muchas veces formales, circunscritos a conceptos operacionales, relativamente sustantivos sobre su contenido y esencia como proceso de la realidad, en los cuales son nucleares argumentos de filiación a partir de teoriza- ciones de alcance medio, sin que se muestren articulaciones con redes más complejas y amplias de teorías. A ello remite Boisier cuando califica de "tautologización" del concepto al sostener que "(...) el desarrollo es precisamente -si se le entiende bien-- exactamente todo aquello que se le atribuye" (Boisier, 1999).

Sucintamente estas consideraciones se pueden agrupar en relación con:

- Cualidades del desarrollo: Expresado en una variedad de producciones teóricas y de prácticas donde el desarrollo se ha venido enunciando en formulaciones tales como social, humano, sostenible, multidimensional, endógeno, ecodesarrollo, autodesarrollo para caracterizar a los objetivos implícitos que, contentivos de diversas finalidades y las vías para alcanzarlos, vienen siendo, aparentemente, expresiones críticas a una noción de desarrollo como progreso lineal y homogeneizante.

\section{- Dimensiones del desarrollo:} Se consideran aquellas posiciones que enfatizan ${ }^{3}$ en las dimensiones económica, social, humana, cultural, etc. in-

3. Los criterios de filiación vía "énfasis" son peligrosos pues de alguna manera reproducen sesgos disciplinares y desarticulan la complejidad social. 
distintamente y, además, aquellas que parten de consideraciones espaciales del desarrollo que se mueven en torno a la espacialidad física y la espacialidad social, entre lo macro y lo micro, lo local y lo global, la sociedad y la comunidad.

Cada una de las tipologizaciones adjudicadas al término desarrollo redundan en el sobredimensionamiento y por ende la subvaloración de aspectos particulares que son parte de un único proceso. Estos "desarrollos" se presentan como un continuum, muchas veces acrítico, con relación a sus contextos de aparición y son traspolados a las circunstancias actuales de existencia social sin que medien procesos de deconstrucción, reelaboración y renovación crítica de las propuestas que, desde las ciencias sociales, se hacen a los decisores.

El neoliberalismo enfocó y focalizó las contradicciones a las localidades, por lo que el desarrollo de las mismas era una meta y una alternativa indispensable para muchos países, sobre todo para los "subdesarrollados". De ahí que al término desarrollo le comience a acompañar lo local, entendido muchas veces en su concepción de espacio despreciando el sistema de relaciones sociales que esencialmente lo compone.

\section{CRÍTICA A LA EMERGENCIA DE}

\section{LO LOCAL}

En nuestra consideración el desarrollo local tiene dos caras: (1) la que señala su rostro creativo y transformador, práctico-crítico a instancias de lo que descubre ausente de la realidad social en movimiento, respecto a lo que falta en el presente para situarlo en la perspectiva de futuro emancipador, universal, donde los vínculos comunitarios de los trabajadores y los oprimidos, se opondrán al marco privado y atomista generados por estas mismas relaciones sociales, y (2) la que se muestra, una vez desembozada, plagada de soluciones emergentes y situacionales, de refuncionalización y conservación del status quo, reproductoras de las injusticias y las contradicciones de la sociedad burguesa (Riera, 2012). 
La racionalidad local a partir de lo planteado y en medio de las ineludibles relaciones nacionales e internacionales, obliga a su sistemización, a estructurarse en una lógica emancipatoria que implique:

1. Lo económico como socioeconómico,

2. La desalienación del trabajo, la realización del productorpropietario real de los medios de producción y las riquezas sociales,

3. El quiebre de lo local como espacio competitivo; fomento de la cooperación no coactiva entre localidades en un continuo nacional,

4. Lo comunitario como medio y finalidad, calificador del desarrollo local, la interpretación de la finalidad, como la adecuación consciente a un fin, atributo de los seres humanos como sujetos de la actividad,

5. La dialéctica de civilización del Estado y politización de la sociedad civil: socialización de las políticas públicas en espacios de decisión arrebatando tal monopolio a burócratas y tecnócratas, desenlace de las asimetrías entre dirigentes y dirigidos; la socialización de los beneficios de las políticas públicas con la maximización del número de beneficiarios de dichas políticas e inversiones; la ciudadanía como fuente de política,

6. La superación crítica del cierre territorial: local, municipal; el encierro sectorial productivo, tecnológico y administrativo,

7. La estimulación de valores de cobertura universal, la ética del trabajo emancipado, la ética socialista frente a la ética del mercado.

De ahí su conformación como guía para la acción revolucionaria de estos tiempos, para la formación de los trabajadores -manuales e intelectuales- como sujetos orgánicos de y para el verdadero desarrollo humano, el emancipador.

Cuando se trata del contenido emancipatorio del desarrollo local la salida teórico-práctica implica comprender que la lógica de la relación mercantil con su accionar hegemónico, no se retrotrae, incluso en los procesos socialista en el siglo XXI, por lo que hay que franquearla a partir de prácticas que le sean antisistémicas para frenar entre otros males el "...excesivo énfasis (maximización) en el interés individual (material) ...y la orientación individualista" (Lebowitz, 2015:61-62); 
estructurando alternativas de desarrollo que sean participativas y cooperadas, donde se produzca para las necesidades de los demás.

Acciones que a su vez sean creadoras y autogestoras en toda su diversidad estructural, en torno a un proyecto comunitario de opciones múltiples opuesto a los recetarios depurados por la inmediatez y a ciertos intereses de poder individualistas y burocratizado que obvian el sentido sistémico de los hechos concretos.

Concebir al desarrollo como proceso de superación de las contradicciones, donde se subordinen las lógicas del capital a la emancipatoria, permite concebir el cambio a partir de la dialéctica entre lo necesario y lo posible. El sistema mundial necesita superar la contradicción capital-trabajo que aliena al hombre y reproduce relaciones sociales asimétricas.

Las diferencias comunitarias que se asocian al efecto del espacio geográfico y sus escalas territoriales, como por ejemplo la oposición entre el centro y la periferia, lo ur- bano y lo rural son el efecto de la distancia en el espacio de relaciones asimétricas, de la distribución desigual de las diferentes especies de capital (económico, cultural, simbólico al decir de Bourdieu) en el espacio geográfico y viceversa, a partir de lo cual comunidad y localidad son escenarios de luchas que no tienen a lo clasista como telón de fondo sino que los cualifica. Las solidaridades prácticas y las rivalidades prácticas tienen su anclaje aquí.

(La)...apropiación del producto objetivado del trabajo social acumulado define el estado de las relaciones de fuerza, institucionalizadas en los status sociales perdurables, socialmente reconocidos o jurídicamente garantizados, entre agentes objetivamente definidos por su posición en esas relaciones; determina los poderes actuales o potenciales en los diferentes campos y las probabilidades de acceso a los beneficios especificos que los campos proporcionan (Bourdieu, 1990:284).

Los sistemas fijos y de flujos son reciprocidades influyentes en la localización territorial de las acciones del desarrollo, en la delimitación de las mismas y de las particularidades de los vínculos co- 
munitarios en los espacios locales de sociedad normada. La desigual disposición de los recursos naturales, los cambios cuantitativos y cualitativos de ellos y de las fuerzas de producción, las relaciones de propiedad, de distribución y consumo, de apropiación, los cambios en la estructura social, las diferencias de la subjetividad, la evolución histórica y la acción política, las relaciones de poder obran sobre la dimensión y el contenido, la planeación, la organización, la participación de los agentes sociales en torno al desarrollo.

Con ello se puede dar cuenta de la variación geográfica y temporal, bajo la lógica de la opresión (del capital), de las relaciones de explotación, de depredación ambiental, de las asimetrías dirigentes-dirigidos, Estado-sociedad civil, del quiebre y la latencia de lo comunitario $y$, a instancias de la lógica de la emancipación social (del trabajo), de un proceso de producción y apropiación social donde el hombre se contempla a sí mismo en el mun- do que ha creado, como ser esencial, lo cual exige la armonización y coherencia de los intereses locales y nacionales, individuales y comunitarios con los de la sociedad, como totalidad en emancipación. Se va verificando el proceso de civilización del Estado y politización de la sociedad civil.

Ambas lógicas transversalizan la estructura espacial y vincular así como las lógicas de regulación horizontal y vertical ${ }^{4}$ por lo que, funcionalmente, lo hacen como un todo.

Se hace necesario entonces develar, hacer consciente el potencial de desarrollo no realizado desde la contradicción contenida en la realidad y ajustar la acción transformadora a las zonas de desarrollo próximo de los grupos sociales en condiciones de asimetría social. Es, entonces, hablar de autodesarrollo o lo que es igual hablar del desarrollo dialécticamente -ya sea local, comunitario, sostenible, etc.- por tanto él:

4. La lógica de regulaciones horizontales, propias de las sociedades territoriales antiguas y tradicionales, no ha desaparecido sino que coexiste con la lógica de regulación vertical, propia, del funcionalismo introducido por la industrialización y la modernización. El desarrollo local para esas sociedades es la forma normal de reproducción social y vuelve, envuelto ahora en un velo tecnológico, a reinstalarse como forma de reproducción social y territorial. 
- Tiene su clave, como proceso, en el sistema de contradicciones sociales.

- Es un proceso contradictorio e histórico concreto de pérdida y recuperación, de realización de la esencia humana.

- Existe como dialéctica de la conservación y el cambio y de la contradicción entre lo viejo y lo nuevo donde se vaya subordinando todos los elementos de la lógica del capital, a través de un proceso de creación, legitimación e institucionalización de una lógica centrada en los seres humanos (Lebowitz, 2008).

- Implica la presencia de lo consciente y lo espontáneo. Muchas veces la acción escapa a nuestras intenciones. La acción supone complejidad, es decir, elementos aleatorios, azar, iniciativa, decisión, conciencia de los alejamientos del fin y de las transformaciones. El triunfo sobre el capital y su lógica está dependiendo de la organización de quienes a él se oponen guiados por el saber, tal y como nos dice Marx desde El Capital.

- Está mediado por la subjetividad. Tiene como momentos la reflexión crítica y el proyecto. La estrategia permite, a partir de una decisión inicial, imaginar un cierto número de escenarios para la acción, escenarios que podrán ser modificados según las informaciones que lleguen en el curso de la acción y según los elementos aleatorios que sobrevendrán y la perturbarán.

- El progreso como dirección del desarrollo, progreso cultural. Articulado en dos dimensiones: la relación de los hombres con la naturaleza (la dimensión técnica) y las relaciones de los hombres entre sí (dimensión social) coincidiendo con Acanda (2002).

- Incluye el desarrollo de la individualidad como auto transformación práctica, vista a través del papel que desempeñan los trabajadores como sujetos colectivos, el desarrollo de las capacidades humanas, vinculándolo con la práctica social y el abordaje de las necesidades sociales y sus satisfactores $^{5}$ (Neef, 1998) desde la jerarquización en su análisis de la perspectiva de lo comunitario (Lebowitz, 2015).

5. "Mientras un satisfactor es en sentido último el modo por el cual se expresa una necesidad, los bienes son en sentido estricto el medio por el cual el sujeto potencia los satisfactores para vivir sus necesidades. Cuando la forma de producción y consumo de bienes conduce a erigir los bienes en fines en sí mismos, entonces la presunta satisfacción de una necesidad empaña las potencialidades de vivirla en toda su amplitud. ... La vida se pone, entonces, al servicio de los artefactos en vez de los artefactos al servicio de la vida. La pregunta por la calidad de vida queda recubierta por la obsesión de incrementar la productividad de los medios."(Neef, 1998 : 35) 
Esto significa que cada individuo participe en la dirección de su producción y reproducción social, porque "(...) las condiciones de la emancipación social e individual no pueden ser separadas, ni mucho menos puesta a enfrentarse entre sí. O prevalecen o fracasan juntas (...) Porque la una necesita enteramente de la otra para poder realizarse" (Mészáros, 2009: 270). Es la esencia comunitaria del desarrollo humano ${ }^{6}$ asumido aquí, particularmente, en los vínculos locales.

\section{RESONANCIA VS “OLVIDO” DEL PENSAR CRÍTICAMENTE SOBRE \\ EL DESARROLLO}

En la base de la comprensión del papel a desempeñar por la intelectualidad de nuestro tiempo, en sus aportaciones sobre el desarrollo, está la superación crítica de la función intelectualista aquella que concibe al mundo como un espectáculo a ser interpretado y no como conjunto de problemas concretos que reclaman soluciones prácticas (Bourdieu y Wacquant, 2005).
Transitar de una concepción tecnocrática de la profesión y del trabajo intelectual a una concepción humanista que recupere la sustantividad valorativa, ética y política de la razón científica en la explicación, comprensión y realización del desarrollo desde su esencia humana liberadora se plantea como acción impostergable no solo para reconocer la fuerza de la reproducción del capital sino también su vulnerabilidad generada por el antagonismo latente entre la producción constante de nuevas necesidades insatisfechas y los salarios mantenidos dentro de los límites de la subsistencia (Lebowitz, 2006).

Desconocer los elementos nucleares de esta transición puede comprometer la superación crítica de las conceptualizaciones y prácticas del desarrollo con la que hemos operado -quedándose a nivel cosmético- limitando el real desarrollo humano contenido en la teoría como instrumento para la acción.

6. "Dentro de la comunidad real y verdadera los individuos adquieren al mismo tiempo su libertad de asociarse y por medio de la asociación" (Marx y Engels, 1975: 82) 
En nuestro criterio tal transición implica:

a) cuestionarnos no sólo, dice Walter Benjamín, cómo una teoría científica declara situarse frente a las luchas sociales por la emancipación, la identidad, la liberación, sino cómo funcionan en las propias luchas (en Žižek, S. 2007).

b) dejar atrás la función de consejeros de príncipes (Munné, F. 1979) en la comprensión del desarrollo, situarlo en el horizonte intelectual de sus reflexiones y actuaciones en relación con su esencia y manifestaciones externas, instrumentales y sustanciales, de apremios y de proyecciones estratégicas, de incertidumbres y certezas.

c) analizar con rigor y enfrentar las proposiciones teóricas y prácticas que nacen desde el mercado, y ponderar a la cultura, asumiéndola como recurso para ponerle freno y hacerlo retroceder en su afán cosificador de los seres humanos y sus relaciones;

d) aprender y enseñar a pensar a partir de cuestionar lo que se ha considerado como una prueba exitosa de una teoría, en un indicador con relación a cuál es el problema, pues ocurre a menudo, que el mecanismo que se adopta para el propósito de la teoría no es necesariamente el mismo que el mecanismo real que produce resultados de acuerdo con la teoría.

Enfrentar el "cientificismo" que reduce el conocimiento a la ciencia, obviando o subestimando los saberes acumulados por la experiencia del vivir cotidianamente de las personas, y la reducción de la ciencia a un surtido de conocimientos que sirven indiferentemente a cualquier fin.

Este proceso crítico tiene que ser de auto-formación profesional permanente, que exija ganar en niveles de reflexividad teórica respecto a la problemática social, sus salidas "desarrolladoras-desarrollistas" y a la producción intelectual que la aprehende, los esquemas que dominan nuestras ciencias y prácticas sociales, las docentes -casi siempre importadas- que no responden a los intereses de justicia social y dignificación humana a la que aspiran, justamente, nuestros pueblos.

Los intelectuales que participamos como maestros, académicos, investigadores, dirigentes, adminis- 
tradores, técnicos en la realización de proyectos sociales de desarrollo -en la producción, reproducción y difusión de valores, modos de vida y actividad, principios de organización del tiempo y el espacio, cultura-, tenemos el compromiso y la doble responsabilidad de no disociar el conocimiento de la vida humana ni de la relación social, los hechos y las decisiones, la razón teórica y razón práctica en la relación medios-fines de un desarrollo humano que nos libere de cualquier forma de opresión, de la trama relacional de supra y subordinación que engloban todas las determinaciones relacionales, consistentes con la configuración de un orden en que unos se someten a otros, y unos acotan las posibilidades de otro.

Es promover modos de actuación y actuar en tanto un sujeto que se posiciona política y críticamente en la realidad y ante sus apariencias. "Pensar al pensamiento y pensar la realidad constituyen por lo tanto dos momentos de un mismo proceso", sintetiza Acanda (2002:5).
Para entrar de lleno como intelectuales orgánicos en los debates sobre el desarrollo en este período de transición o reemergencia crítica (Espina, 2004) de dicho término, y sus derivaciones para la praxis transformadora, no es necesario sucumbir en un campo de batallas especulativo; se trata de producir una comprensión del desarrollo que permita, como propuesta teórica orientadora, co-construir las alternativas de cambio del orden existente y que sirva a los fines de una práctica social cualitativamente superior frente a otras que se presentan como producciones algorítmicas, como esquema a imponerle a la realidad, considerando a las personas de modo unilateral y por consiguiente, la oportunidad de desarrollo que se les brinda, mutila la capacidad creativa para su desenvolvimiento pleno y multilateral.

La oportunidad de contribuir decisivamente, desde la actividad que desplegamos, está en primera instancia, en la auto-concienciación de nuestras limitaciones y déficit como intelectuales y docentes, partiendo 
de lo cual podamos, a través de una honda y profunda (auto) crítica de nuestras construcciones analíticas y nuestras prácticas profesionales, no postergar a la teoría con la obsesión de la impecabilidad metodológica e instrumental (Bourdieu, 1999).

La experiencia y la información acumulada deben re-significarse en un pensamiento que no puede quedarse simplemente al nivel de la realidad existente, de lo urgente, de lo empírico y lo práctico contingente.

La contradicción teoría y práctica es dialéctica.

...los hombres que producen las relaciones sociales con arreglo a su productividad material ... crean también las ideas y las categorias, ... las expresiones ideales abstractas de esas mismas relaciones sociales ...La abstracción, la categoria, considerada como tal, es decir, separada de los hombres y de su acción material, es, naturalmente, inmortal, inalterable, impasible; no es más que una modalidad de la razón pura, lo cual quiere decir, simplemente, que la abstracción, considerada como tal, es abstracta: itautologia maravillosa! (Marx, C. 1846:7-8).

La categoría desarrollo sólo dejará de ser una modalidad de la razón pura cuando se supere el polo teórico de esta relación dialéctica, con la potencialidad que despliegue esta idea en la práctica transformadora (Sánchez Vázquez, 2004:500).

El pensamiento crítico, renovador sobre el desarrollo, como síntesis de múltiples determinaciones, debe abarcar alternativas de modo creativo en la planeación del futuro. Esta oportunidad requiere la deconstrucción del positivismo como teoría y cultura, de no hacerlo así, reforzaríamos el statu quo que lo perpetua.

La labor que nos compete, en este orden, consiste en movilizar nuestra capacidad de pensar para formular conceptos y categorías, teorías y argumentos, que puedan contribuir a realizar un cambio social humanizador mas, la acepta- 
ción y legitimación de la teoría revolucionaria que se ponga a disposición de dicho cambio social, dependerá de la energía puesta en la práctica revolucionaria que también nos pertenece.

Por tanto a partir de ello la propuesta es crítica y proyectiva tratando de alejarnos en nuestra práctica profesional del criticismo académico y el instrumentalismo pragmático. De ahí que:

- En las conceptualizaciones de desarrollo que se asuman tienen que quedar sustantivamente fijados los objetivos de la emancipación como proceso histórico y concreto, en sus gradaciones.

- En la formulación del mismo, o se da cuenta de la realidad a transformar, desde el "lado del trabajo" y sirve como instrumento para el proceso del reencuentro de la humanidad con su esencia o, explícitaimplícitamente, se pone del "lado del capital" y su sentido alienador-cosificador-manipulador-fragmentador.

- Se hace necesario descubrir tras cada concepción del desarrollo la concepción del ser humano que entraña, y descubrir a quienes se consideran como tal.
La labor y el compromiso de los intelectuales, dentro y fuera de la academia, está en deconstruir para contribuir a desaprender aquellas posiciones, que explícita o implícitamente, conscientes $o$ ingenuamente, tienden a la refuncionalización como única alternativa de cambio de la realidad social amén de que en su presente estén refundidas las premisas de un permanente proceso de transformación revolucionaria.

Remitimos desde aquí a una voluntad que tendrá que sustraerse a la fuerza manipuladora y condicionante de la reproducción de lo existente, a la fuerza de absorción de la razón instrumental, promoviendo sujetos implicados en la lucha los cuales, obcecados por los árboles, no pierdan de vista el bosque teniendo en cuenta, por demás, no olvidar a los árboles.

A partir de ello es que la idea de desarrollo asociado a lo territorial nos revela la necesidad de situarlo en la perspectiva del contenido de dicho proceso en las diferentes escalas espaciales de producción de la dinámica social. La comunidad y 
la localidad en íntima y orgánica relación estructuran y son estructuradas por lo vincular y lo espacial respectivamente, concentrando las propiedades de la sociedad concreta como totalidad y en su contrariedad: lo dado y lo potencialmente por darse.

Es un hecho empíricamente comprobable que la cuestión territorial, local, regional tiene siempre bases materiales, entendidas como el proceso del trabajo, de la apropiación de la naturaleza por el hombre organizado socialmente, las relaciones de producción -aunque estas sean mínimas- el metabolismo social, elementos a los que hay que recurrir para descifrar las bases de las estructuras conformadas espacialmente y para comprender que en los espacios locales se verifican interrelaciones más amplias del tejido social histórico, permitiendo incluso develar la presencia de las relaciones cooperadas y solidarias como elementos de lo nuevo a promover y reproducir si de desarrollo emancipador se habla. Ello remite a la escala geográfica de un proceso sustantivo.
La distribución de poderes está presente en el espacio geográfico sobre la base de la acotación en un modo preciso de posición, de status $y$ de regiones determinadas de dicho espacio como un conjunto de relaciones de fuerzas objetivas que no se pueden reducir a las intenciones de los agentes individuales y a las interacciones directas entre los agentes (Bourdieu, 1990). Desde la situación económica hasta el prestigio y la reputación, en este juego de fuerzas, se constituyen los intereses y las posiciones de los agentes del cambio social transversalizado por la idea de cambio en y/o cambio de una sociedad.

\section{POSIBILIDAD DE UN}

\section{DESARROLLO HUMANO LOCAL: CONCLUSIONES}

Siguiendo la lógica de los argumentos aportados, el desarrollo local debe dejar de ser concebido como homólogo de modernización desde el determinismo tecno-económico, para centrarse en una gestión del Estado y el gobierno nacional y local que privilegie: (1) la potenciación del trabajo por encima de la búsqueda de nichos de mercado, al 
interior y al exterior y (2) una socialización comunitaria, desde un análisis sereno de la realidad concreta, lo que implica desafíos de posibilidad.

En los discursos de desarrollo que contienen la incontrastable rebeldía de las mayorías en relaciones de opresión, demandantes de un cambio de su situación se constata la ponderación de la participación popular como medio eficaz para facilitar e influir en los procesos de cambio a su favor.

Sin embargo enunciar la participación no es el ¡Abracadabra! de un desarrollo humano, alternativo, viable y sostenible frente a la lógica de la opresión y a los procesos de reproducción de sus hegemonías; es necesario tener en cuenta los condicionamientos para una participación real y diferenciada.

Si ésta no se produce como medio de una socialización y activismo comunitario que realice en la práctica rearticulaciones entre los oprimidos por cualquier condición, puede estar como elemento refuncionalizador de las hegemonías alienadoras.
Develar las fórmulas del populismo, de la despolitización de los problemas de una sociedad, es una tarea de importancia táctica y estratégica frente a los discursos vacíos y manipuladores de la participación como vehículo "neutral" del desarrollo local.

Las salidas locales a problemáticas universales, que cumplan con "...compartir el patrimonio social de los seres humanos... para poder desarrollar su pleno potencial" y que estos junto a las capacidades se desplieguen "...mediante democracia, participación y protagonismo en el centro de trabajo y la sociedad" en "...comunidades basadas en la cooperación y la solidaridad" (Lebowitz, 2015:114) permite rescatar al desarrollo local como concepción y práctica orientada al cambio emancipador.

Lo comunitario en su sentido de cualidad puede proveer el sustrato humanista desalienador al pensamiento y la práctica sobre el desarrollo local ya que "... solo puede emanar directamente de las acciones, aspiraciones, y conciencia 
creativa y crítica de los propios actores sociales, que de ser tradicionalmente objetos del desarrollo, pasan a asumir su rol protagónico de sujetos." (Neef, 1998:67).

Es en este proceso donde el autodesarrollo de cada individuo puede interpretarse como la supresión de toda clase de barreras que impiden a una persona determinada, miembro de la comunidad en cuestión y habitante de la localidad y del Estado-Nación, alcanzar su plena realización como persona humana, en este entramado de redes de relaciones del nosotros con los otros y no a expensas de ellos, del ciudadano-hombre reconquistado.

A partir de aquí, el debate sobre el desarrollo, la cuestión de lo local y lo comunitario, no es solo académica sino fundamentalmente política e ideológica por tanto la intelectualidad orgánica es corresponsable junto a los pueblos de pensarlo y actuarlo orgánicamente. De ahí la necesidad de recuperar el valor de la teoría como crítica práctica de lo existente, como interpelación de la realidad y de los conflictos que la atraviesan.
Para el pensamiento crítico -que es el de la emancipación humana, el enfrentado a las formas de dominación social (León del Río, 2010)un reto está en capturar la necesidad de una práctica consecuente con un desarrollo local verdaderamente humano, en la misma medida que da cuenta de una realidad que necesita ser renovada sistémica y sistemáticamente.

\section{BIBLIOGRAFÍA}

Acanda J. L. (2002): ¿Qué significa ser progresista en materia de pensamiento? (Publicado en: Hacia dónde va el pasado. El provenir de la memoria en el mundo contemporáneo. Barcelona, Paidós, Entregado a filosofi@.cu por el autor).

Becerra, F.; Pino, J. (2005) Evolución del concepto de desarrollo e implicaciones en el ámbito territorial: experiencia desde Cuba. Economía, Sociedad y Territorio, $\mathrm{V}$.

Boisier, S. (1999) Desarrollo (local): ¿De qué estamos hablando? [En línea] [http://www.cedet.edu.ar/sitio/administracion/agenda/boisier.pdf Consultado el 12-6-2003]

Bourdieu P. et al (1999) El oficio del sociólogo. Siglo XXI Editores.

Bourdieu, P. (1990) Sociología y Cultura. México, Editorial Grijalbo, S.A.

Bourdieu y Wacquant, (2005) Una invitación a la sociología reflexiva. Siglo XXI Editores. Argentina, http://www.scribd.com/insurgencia [Consultado el 3-7-2006] 
Espina Prieto, M. (2004) Humanismo, totalidad y complejidad. El giro epistemológico en el pensamiento social y la conceptualización del desarrollo. En La participación. Diálogo y debate en el contexto cubano. Centro de Investigación y Desarrollo de la Cultura Cubana Juan Marinello. La Habana

Féliz, M. (2015) ¿Qué hacer...con el desarrollo? Neodesarrollismos, buenvivir y alternativas populares. Revista Sociedad y economía No. 28 , pp. 29-50

Lebowitz, M. (2006) Más allá de El Capital. Monte Ávila, Caracas

Lebowitz, M. (2008) El socialismo no cae del cielo: un nuevo comienzo. Monte Ávila, Caracas

Lebowitz, M. (2015) La alternativa socialista: el verdadero desarrollo humano.

Editorial Ciencias Sociales. La Habana

León del Río, Y. (2010) "El pensamiento crítico en pos de la emancipación humana. Fundar políticas, éticas y simbólicas sin exclusiones". Biblioteca Virtual de Filosofía y Pensamiento Cubanos. http://biblioteca.filosofia.cu/. Cuba.

Marx C. y Engels, F. (1975) La Ideología Alemana. Editora Política, La Habana.

Marx, C. (1846) Carta a P. V. Annenkov del 28/12/1846. Versión digital en http:Ilwww.marxist.org

Max-Neef, M. et al (1998) Desarrollo a escala humana, conceptos, aplicaciones y algunas reflexiones. Icaria. Barcelona.

Merton, R. (2002): Teoría y estructura social. Política / Teoría Social / Teoría Sociológica II. Sobre las teorías sociológicas de alcance intermedio. (Pdf) Biblioteca CEC-UCLV. Cuba
Meszáros, I. (2009) Más allá del capital. Hacia una teoría de la transición. Editores Vadell Hermanos, Caracas.

Munné, F. (1979) Prólogo al libro Grupos, masas y sociedad. Introducción sistemática a la sociología general y especial

Sánchez -Vázquez, A. (2004) A Tiempo y destiempo. Antología de ensayos. Editorial. Ciencias Sociales, La Habana.

Pérez Díaz, A. (2010) Desarrollo local: Estudio sobre las condiciones del gobierno para generar desarrollo local en el municipio de Manicaragua, provincia de Villa Clara. Tesis en opción al Grado Científico de Doctor en Ciencias Sociológicas, Cuba

Riera, et al (2017) Una aproximación al desarrollo desde la concepción comunitaria. Editorial Feijóo. Villa Clara, Cuba

Riera, C. M. (2012) Lo comunitario como cualidad del desarrollo local. Su alcance práctico para la transformación emancipadora de la sociedad. Tesis presentada en opción al grado científico de Doctor en Ciencias Sociológicas. Cuba

Žižek, S. (2007) Recordando a Lenin. Rebeliones. Editorial Ciencias Sociales. La Habana.

Zemelman, H. (1987) Conocimiento y sujetos sociales. Contribución al estudio del presente. El Colegio de México, México D.F. 